\title{
Higher levels of mucosal antibody to pneumococcal vaccine candidate proteins are associated with reduced acute otitis media caused by Streptococcus pneumoniae in young children
}

\author{
$\mathrm{Q} \mathrm{Xu}{ }^{1}$, JR Casey $^{2}$ and ME Pichichero ${ }^{1}$
}

Mucosal immunity has a crucial role in controlling human respiratory tract infections. This study characterizes the naturally acquired mucosal antibody levels to three Streptococcus pneumoniae (Spn) protein antigens, pneumococcal histidine triad protein $D(P h t D)$, pneumococcal choline binding protein $A(P c p A)$, and pneumolysin (Ply), and assesses the association of the mucosal antibody levels with occurrence of acute otitis media (AOM) caused by Spn. Both nasopharyngeal (NP) immunoglobulin G (IgG) and IgA levels to all three proteins slightly decreased in children from 6 to 9 months of age and then gradually increased through 24 months of age. Spn NP colonization was associated with higher mucosal antibody levels to all three proteins. However, children with Spn AOM had 5-8-fold lower IgG and 3-6-fold lower IgA levels to the three proteins than children without AOM but asymptomatically colonized with Spn. Antigen-specific antibody levels in the middle ear fluid (MEF) were correlated with antibody levels in the NP. Children with AOM caused by Spn had lower antibody levels in both the MEF and NP than children with AOM caused by other pathogens. These results indicate that higher naturally acquired mucosal antibody levels to PhtD, PcpA and Ply are associated with reduced AOM caused by Spn.

\section{INTRODUCTION}

Streptococcus pneumoniae $(S p n)$ is responsible for a large spectrum of infectious diseases in children and adults such as sepsis, pneumonia, meningitis, sinusitis, and acute otitis media $(\mathrm{AOM}){ }^{1-3}$ To date, 94 distinct serotypes have been documented based on capsular composition. ${ }^{3,4}$ Current licensed 23-valent pneumococcal polysaccharide vaccine (PPV-23) and 7-, 10-, and 13-valent pneumococcal conjugate vaccines (PCV-7, -10, -13) significantly reduce invasive pneumococcal diseases, ${ }^{1,5}$ but coverage is limited to the vaccine serotypes. After introduction of PCV-7, and more recently PCV-13, there has been a decrease in carriage caused by vaccine serotypes but an emergence of non-vaccine replacement serotypes. ${ }^{1,3,6-8}$ Furthermore, current polysaccharide-based vaccines are less effective in non-invasive pneumoniae and $\mathrm{AOM}^{9}{ }^{9,10}$ In addition, PPV-23 is currently recommended in the elderly and high-risk adults. It is poorly immunogenic in children, especially $<2$ years of age. ${ }^{11}$ Because of these shortcomings, protein-based vaccine candidates have been sought to replace or complement current polysaccharide-based vaccines. ${ }^{9,12,13}$

A number of pneumococcal protein antigens have been studied as vaccine candidates against pneumococcal infection, and multiple proteins have shown sufficient promise to enter human clinical trials. ${ }^{12,13}$ Monovalent protein vaccine candidates of PhtD (pneumococcal histidine triad protein $\mathrm{D}$ ), ${ }^{14}$ PcpA (pneumococcal choline binding protein A), ${ }^{15}$ PspA (pneumococcal surface protein A), ${ }^{12}$ and dPly (detoxified pneumolysin), ${ }^{16}$ bivalent protein vaccine candidates $\mathrm{PhtD} /$ PcpA, ${ }^{15} \mathrm{PspA} / \mathrm{PsaA}$ (pneumococcal surface adhesin A), ${ }^{12} \mathrm{PhtA} /$ $\mathrm{PhtB},{ }^{12}$ and PhtD/Ply alone or along with PCV-7, (ref 12) and trivalent protein vaccine candidates $\mathrm{PhtD} / \mathrm{PcpA} / \mathrm{PlyD} 1$ and ${ }^{12} \mathrm{PhtD} / \mathrm{Ply}$ with protein D of Haemophilus influenzae ${ }^{13}$ were or are in human clinical trials. Our laboratory has been studying three pneumococcal proteins-PhtD, PcpA, and

${ }^{1}$ Rochester General Hospital Research Institute, Rochester, New York, USA and ${ }^{2}$ Legacy Pediatrics, Rochester, New York, USA. Correspondence: Q Xu, (Qingfu.xu@rochesterregional.org)

Received 18 June 2014; accepted 24 December 2014; published online 4 February 2015. doi:10.1038/mi.2015.1 
PlyD1 (genetic detoxified pneumolysin), which are the components of a candidate vaccine in a trial sponsored by Sanofi Pasteur. PhtD is a divalent cation-regulated surface protein, shown to elicit protection against nasopharyngeal (NP) colonization, and is highly conserved across pneumococcal serotypes. ${ }^{17} \mathrm{PcpA}$ is another divalent cation-regulated surface protein that has a major role in pneumococcal adherence. ${ }^{15}$ Both PhtD and PcpA have been shown to be involved in adherence of Spn to human NP epithelial cells in vitro. ${ }^{18,19} \mathrm{Ply}$ is a cholesterol-dependent secreted cytolysin, which is a key virulence factor contributing to bacterial pathogenesis at both early and late stages of infection. ${ }^{16}$ In mice studies, immunization with PhtD, PcpA, and PlyD1 have been shown to elicit protection. ${ }^{20-24}$ In our previous studies, we have detected specific antibodies to PhtD, PcpA, and PlyD1 antibodies in children from natural exposure to $S p n$ in sera after NP colonization and $\mathrm{AOM} .^{25}$ However, upon further analysis, no correlation between serum antibody titers to these proteins and protection of occurrence of Spn AOM could be identified (unpublished data). We therefore hypothesize that mucosal immunity has a critical role in the control of pneumococcal mucosal diseases, such as AOM, sinusitis, and non-bacteremic pneumonia.

Although Spn NP colonization is a necessary prerequisite for infections to develop, carriage is mostly asymptomatic. ${ }^{10}$ However, when the condition of the host is altered, such as by an upper respiratory viral infection, $S p n$ may cause AOM. ${ }^{26}$ Unfortunately, the human mucosal immune response against pneumococci ${ }^{10}$ and to pneumococcal proteins after natural Spn exposure and AOM is poorly understood. In the present study, we characterized the induced mucosal antibody levels in the NP to PhtD, PcpA, and PlyD1 and assessed the association of these antibody responses with the occurrence of natural Spn AOM infections in children aged 6-24 months. In addition, in a previous study, we found middle ear fluid (MEF) antibody in humans originates predominantly from sera and NP secretions. ${ }^{27}$ Here we assessed the correlation of antibody levels in NP secretions with MEF.

\section{RESULTS}

\section{Study cohort}

This analysis involved a total of $424 \mathrm{NP}$ and $152 \mathrm{MEF}$ samples collected during 234 health and 208 AOM visits from 176 children between the ages of 6 and 24 months. One hundred and thirty-three $(76 \%)$ children had both health and AOM visits and $43(24 \%)$ children had only AOM visits. The characteristics of the children are shown in Table 1. As our group has previously shown age-related differences in serum antibody response to the studied antigens, ${ }^{25,28}$ the NP and MEF samples for this study were age matched.

\section{Natural acquisition of mucosal antibody in the NP over time}

First, we evaluated the natural acquisition of NP mucosal antibody in $233 \mathrm{NP}$ samples from 55 children who had at least 4 sequential health visits during the study period. Figure 1
Table 1 Characteristics of children $(n=176)$

\begin{tabular}{lc}
\hline & Number (\%) \\
\hline Female & $80(45 \%)$ \\
Male & $96(55 \%)$ \\
Breast feed & $55(31 \%)$ \\
Formula & $67(38 \%)$ \\
Both & $54(31 \%)$ \\
Smoke exposure & $13(7 \%)$ \\
Non-smoke exposure & $163(93 \%)$ \\
Daycare & $70(40 \%)$ \\
Non-daycare & $106(60 \%)$ \\
PCV-7 up-to-date & $176(100 \%)$ \\
\hline
\end{tabular}

Abbreviation: PCV-7, 7-valent pneumococcal conjugate vaccine.

shows the geometric means (GM) of antibody levels to PhtD, PcpA, and PlyD1 in the NP of children aged 6, 9, 12, 15, 18, and 24 months. Both IgG and IgA antibodies to all three Spn proteins decreased from 6 to 9 months and then increased steadily over time with a peak at 18-24 months of age. The NP antibody levels were associated with age for both IgG and IgA.

\section{Mucosal antibody levels in the NP of Spn-colonized vs. non- colonized children}

Next, we sought to determine differences in detectable antibodies in nasal secretions in $S p n$-colonized vs. noncolonized children. It is well established that $S p n$ colonization in children occurs in the first 6 months of life. ${ }^{29}$ Therefore, we anticipated that detectable mucosal antibodies would be present at the first sampling of our study cohort at 6 months of age. Our hypothesis was that detectable Spn colonization would result in higher mucosal IgG and IgA antibody levels to the pneumococcal proteins. We compared IgG and IgA levels to PhtD, PcpA, and PlyD1 in NP samples from children who had $S p n$ detected in their NP secretions at the time of sampling vs. those who were Spn culture-negative. Children with $S p n$ had significantly higher GM of specific IgG and IgA antibody in the NP than Spn culture-negative children (Figure 2). There was a fivefold higher GM of IgG anti-PhtD (4.39 vs. $0.87 \mathrm{ng} \mathrm{g}^{-1}, P<0.0001$ ), a fivefold higher $\mathrm{GM}$ of $\mathrm{IgG}$

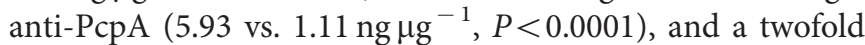

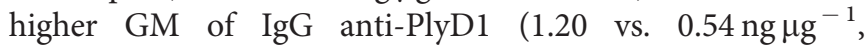
$P=0.001)$. The NP IgA levels to PhtD, PcpA, and PlyD1 were higher by twofold (11.38 vs. $\left.4.67 \mathrm{ng} \mathrm{\mu g}^{-1}, P<0.0001\right)$,

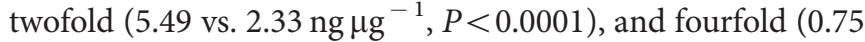
vs. $\left.0.19 \mathrm{ng} \mu \mathrm{g}^{-1}, P=0.0003\right)$ in children colonized with $S p n$ vs. non-colonized, respectively.

\section{Higher mucosal GM of antibody levels in the NP are associated with reduced AOM caused by Spn}

We next investigated whether there is an association of mucosal antibody levels with development of Spn AOM. We compared antibody levels with the pneumococcal proteins in the NP between children with Spn AOM and healthy children asymptomatically colonized with $S p n$. We found that children 

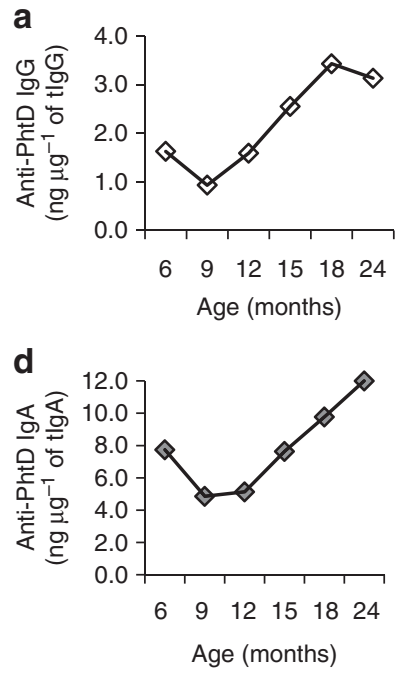
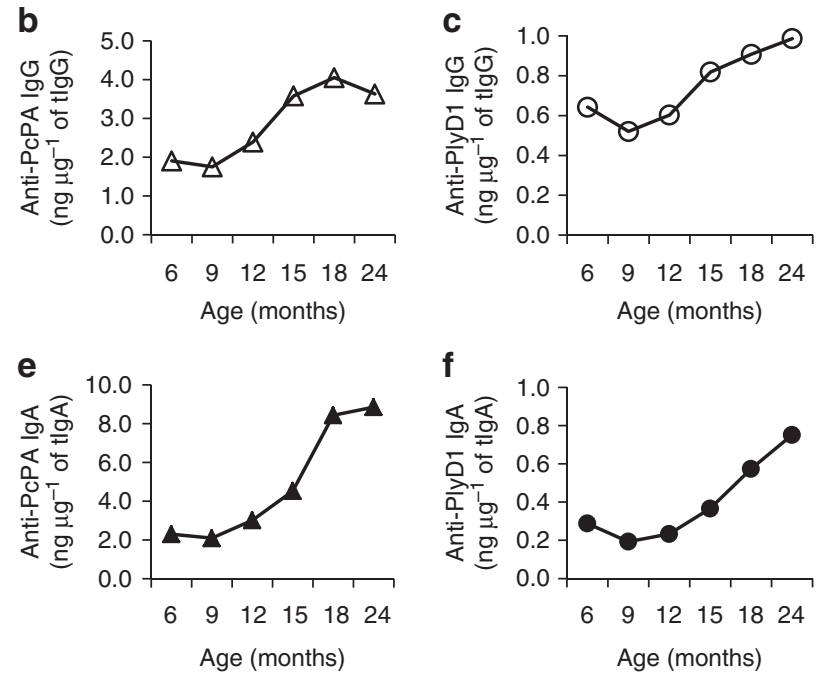

Figure 1 Natural acquisition of nasopharyngeal (NP) mucosal antibody over time. NP samples were collected from children who had at least four regular perspective visits to determine the rates of pneumococcal specific to total immunoglobulin $\mathrm{G}(\lg \mathrm{G})$ and $\lg \mathrm{A}$. Antibody levels were expressed as geometric means of the ratio of specific to total antibody, and association between age and antibody levels was analyzed using repeated-measures logistic regression. $N=47,45,38,35,33$, and 34 for $6,9,12,15,18$, and 24 months of age, respectively. (a), Anti-PhtD IgG; (b), Anti-PcpA IgG; (c), Anti-PlyD1 IgG; (d), anti-PhtD IgA; (e), Anti-PcpA IgA; (f), Anti-PlyD1 tlgA; tlgG and tlgA, total amount of IgG and $\lg A$.
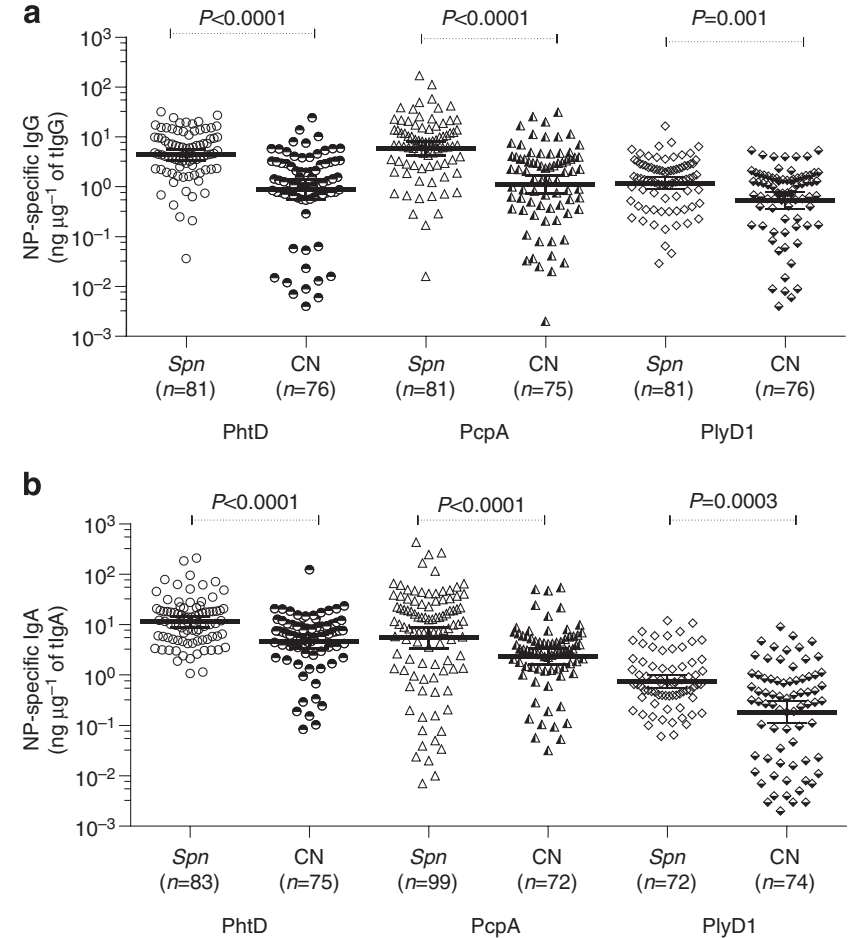

Figure 2 Colonized-children by Streptococcus pneumoniae (Spn) had higher mucosal geometric means (GM) of antibody levels than non-colonized children. Nasopharyngeal (NP) samples were collected from age-matched healthy children with or without Spn colonization. The rates of pneumococcal specific to total immunoglobulin G (IgG) and total IgA were determined by enzyme-linked immunosorbent assay and compared using the nonparametric Mann-Whitney test between culture positive and negative for Spn. (a), Anti-PhtD, anti-PcpA, and anti-PlyD1 lgG; (b), anti-PhtD, anti-PcpA, and anti-PlyD1 IgA; CN, culture negative; tlgG and tlgA, total amount of IgG and IgA. Lines represent GM with 95\% confidence intervals.

who developed Spn AOM had significantly lower mucosal IgG and IgA levels in their NP to all three studied Spn proteins compared with Spn-colonized children who did not progress to
AOM (Figure 3a,b). The NP GM of antibody levels of children with Spn AOM were eightfold lower in anti-PhtD IgG (0.56 vs. $\left.4.39 \mathrm{ng} \mu \mathrm{g}^{-1}, P<0.0001\right)$, fivefold lower in anti-PcpA IgG (1.18 vs. $5.93 \mathrm{ng} \mathrm{\mu g}{ }^{-1}, P<0.0001$ ), fourfold lower in anti-PlyD1 IgG ( 0.29 vs. $1.19 \mathrm{ng} \mathrm{\mu g}^{-1}, P<0.0001$ ), fourfold lower in anti-PhtD

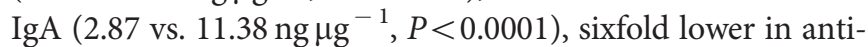
PcpA IgA (0.97 vs. $\left.5.49 \mathrm{ng} \mathrm{gg}^{-1}, P<0.0001\right)$, and threefold lower in anti-PlyD1 IgA (0.28 vs. $\left.0.75 \mathrm{ng} \mathrm{\mu g}^{-1}, P=0.001\right)$ compared with those of healthy children. GM of antibody levels in NP were negatively associated with occurrence of Spn AOM in anti-PhtD IgG (odds ratio $(\mathrm{OR})=0.04,95 \%$ confidence interval (CI) $0.02-0.09, P<0.0001)$, anti-PcpA $\operatorname{IgG}(\mathrm{OR}=0.06$, 95\% CI 0.03-0.12, $P<0.01)$, anti-PlyD1 IgG $(\mathrm{OR}=0.24,95 \%$ CI $0.15-0.37, P=0.0001)$, anti-PhtD IgA $(\mathrm{OR}=0.03,95 \% \mathrm{CI}$ $0.01-0.06, P<0.0001)$, anti-PcpA IgG $(\mathrm{OR}=0.02,95 \% \mathrm{CI} 0.00$ $0.07, P<0.0001)$, and anti-PlyD1 IgG $(\mathrm{OR}=0.1395 \% \mathrm{CI}, 0.07-$ $0.24, P=0.0008)$.

A similar association was found in the NP GM of antibody levels between children with AOM caused by Spn and children with AOM caused by other pathogens. At the onset of AOM, when co-colonization in the NP of Spn with other potential pathogens occurs, the children with Spn AOM have slightly lower GM of IgG but significantly lower GM of IgA to all three pneumococcal proteins than children with non-Spn AOM (Figure 3c,d). Compared with children with non-Spn AOM, children with Spn-AOM had twofold lower GM of anti-PhtD IgG (0.57 vs. $1.10 \mathrm{ng} \mathrm{\mu g}^{-1}, P=0.08$ ), twofold lower GM of anti-PcpA IgG (1.18 vs. $\left.2.82 \mathrm{ng}_{\mu g^{-1}}, P=0.02\right)$, and twofold

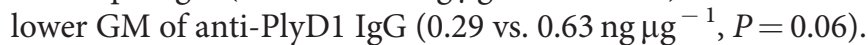
The GM of IgA in the NP of children with Spn AOM

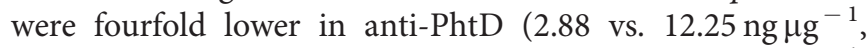

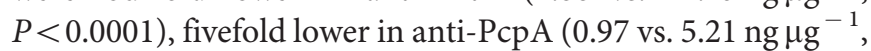
$P<0.0001)$ and threefold lower in anti-PlyD1 $(0.28$ vs. $\left.0.80 \mathrm{ng} \mathrm{\mu g}^{-1}, P=0.001\right)$. 

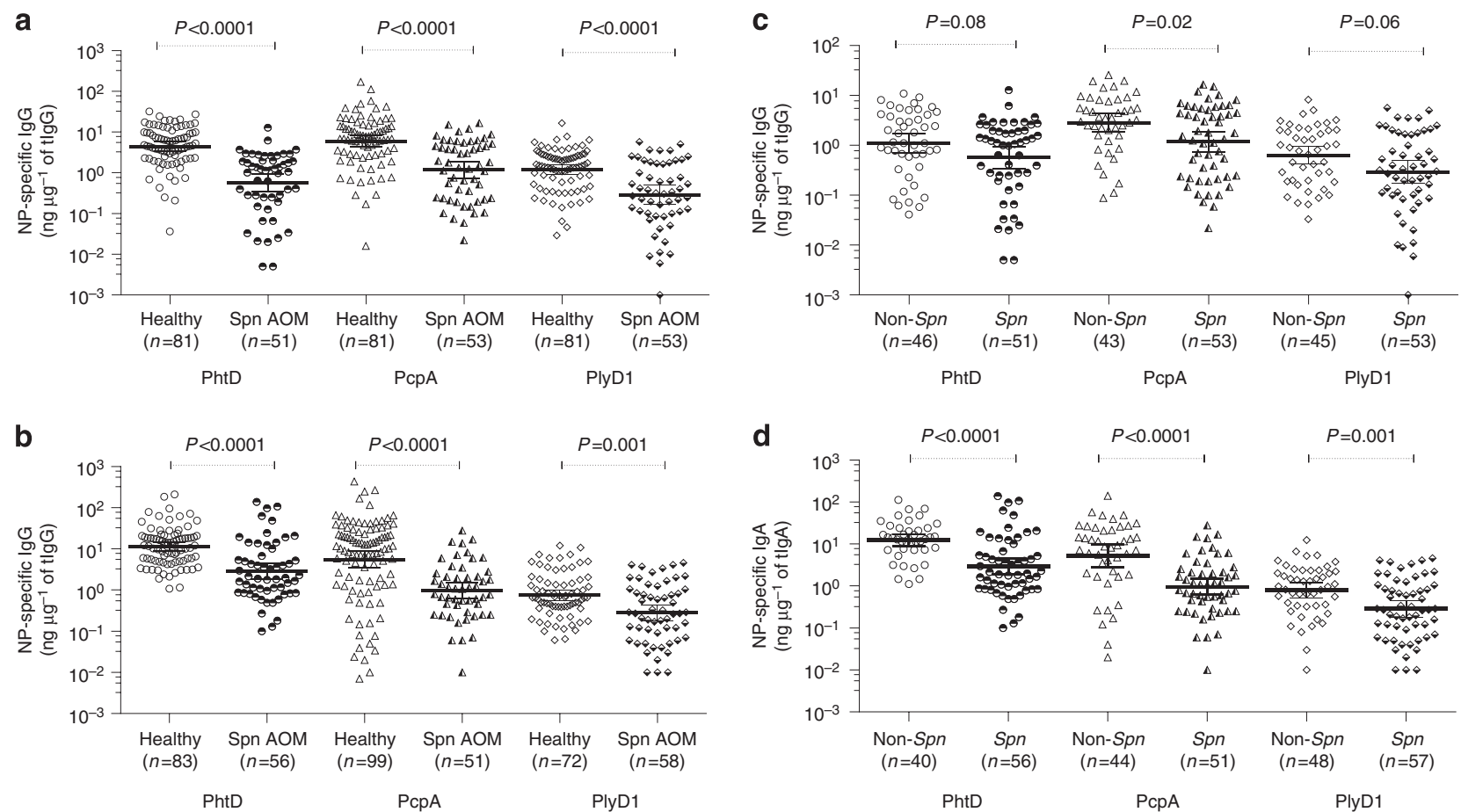

Figure 3 Higher mucosal antibody levels in the nasopharyngeal (NP) are associated with reduced acute otitis media (AOM) caused by Streptococcus pneumoniae (Spn). NP samples were collected from age-matched healthy Spn-colonized asymptomatic children and children with AOM. The levels of pneumococcal specific to total immunoglobulin $\mathrm{G}(\mathrm{IgG})$ and $\operatorname{lgA}\left(\mathrm{ng} \mathrm{\mu g^{-1 }}\right)$ were compared $(\mathbf{a}$ and $\mathbf{b})$ between children with Spn AOM and children with asymptomatic Spn colonization and (c and d) between children with Spn AOM and children with non-Spn AOM using the Mann-Whitney test. (a and $\mathbf{c}$ ): Anti-PhtD, anti-PcpA, and anti-PlyD1 lgG; (b and d): anti-PhtD, anti-PcpA, and anti-PlyD1 IgA; tlgG and tlgA, total amount of IgG and IgA. Lines represent geometric means with $95 \%$ confidence intervals.

\section{Children with Spn AOM have lower GM of antibody levels in the MEF than children with non-Spn AOM}

We also examined the MEF antibody levels to the three studied Spn proteins in children with AOM. At the onset of AOM, children with Spn AOM, compared with children who experienced AOM caused by other otopathogens, had twofold \left. lower GM of anti-PhtD IgG (0.47 vs. $1.64{\mathrm{ng} \mu^{-1}}^{-1}, P=0.0002\right)$, fivefold lower GM of anti-PcpA IgG (0.63 vs. $3.37 \mathrm{ng} \mathrm{\mu g}^{-1}$, $P=0.001)$, and threefold lower GM of anti-PlyD1 IgG (0.17 vs.

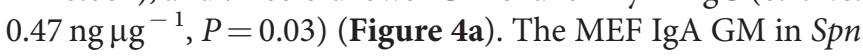
AOM children were threefold lower in anti-PhtD (1.87 vs.

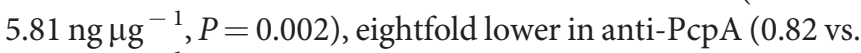
$\left.6.17 \mathrm{ng} \mathrm{\mu g}^{-1}, P<0.0001\right)$ and fourfold lower in anti-PlyD1 (0.2 vs. $0.9 \mathrm{ng} \mu \mathrm{g}^{-1}, P<0.0001$ ) (Figure $4 \mathrm{~b}$ ).

\section{Correlation between antibody levels in the NP and antibody levels in MEF at AOM}

Antibody in NP secretions refluxes from the NP through the Eustachian tube to the middle ear during viral upper respiratory infections. We have previously shown that MEF antibody originates predominantly from sera and NP secretions. ${ }^{27}$ Therefore, we selected available paired NP and MEF samples simultaneously collected from the same children to analyze the correlation between antibody levels in the NP and MEF. Because antibodies were found to correlate significantly with age, to control potential spurious latent correlation, antibody levels were adjusted for age. Both IgG and IgA levels to all three
Spn antigens in the NP were significantly and positively correlated with those in MEF of children at the onset of AOM (all $P$-values $<0.01)$ (Figure 5).

\section{DISCUSSION}

Mucosal immunity is thought to have a crucial role in the control of respiratory tract infections. ${ }^{30}$ Here we address gaps in the knowledge of immune response mounted by children who experience Spn NP colonization and AOM. We characterized the natural acquisition of mucosal antibody to Spn candidate vaccine proteins in young children who experience $S p n$ colonization and consequent AOM. IgG and IgA antibody levels to PhtD, PcpA, and PlyD1 in nasal secretions increased over time, suggesting that sequential natural exposure to $S p n$ results in boosting antibody in mucosal NP secretions. We found that Spn colonization of the NP was associated with higher mucosal antibody responses to the vaccine candidate antigens studied vs. non-Spn colonized NP samples. The results are consistent with previous observations regarding serum antibody responses to the same antigens. ${ }^{25,31}$ Most importantly, we found that children who developed Spn AOM had lower levels of IgG and IgA in nasal secretions at the onset of AOM compared with the antibody levels of children who were NP colonized with Spn but did not progress to AOM infection. Furthermore, when children developed Spn AOM they had lower IgG and IgA levels in their MEF to all three studied proteins compared with children who experienced AOM 
caused by other otopathogens. These results suggest an association between higher NP antibody levels to Spn proteins and a significantly reduced risk of Spn AOM.
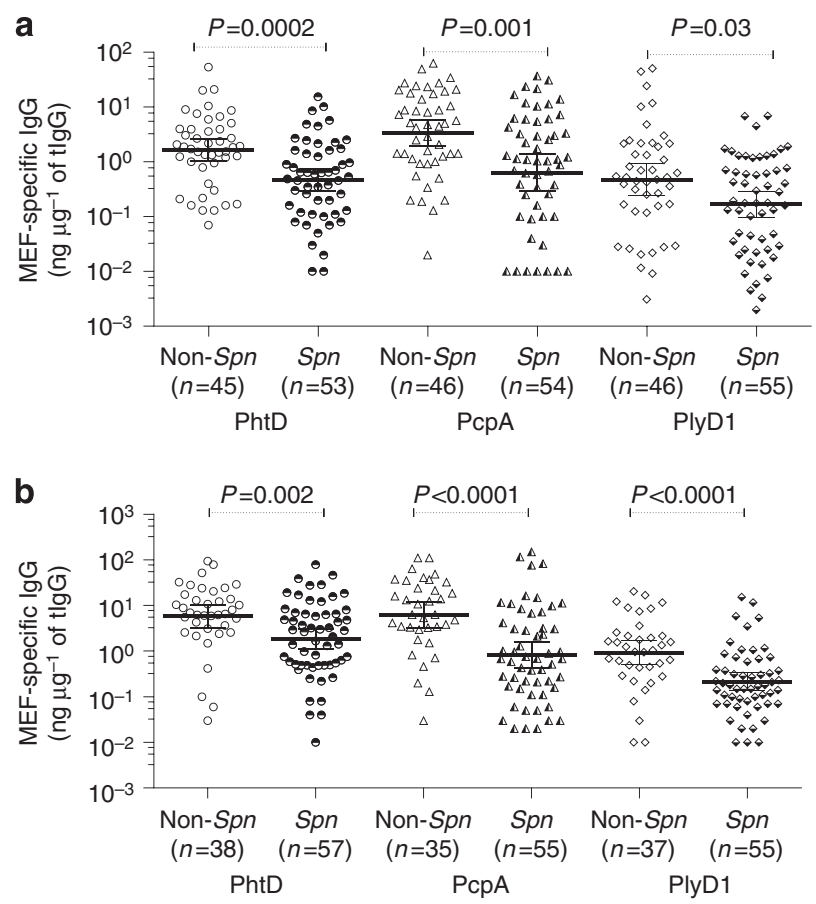

Figure 4 Higher mucosal antibody levels in the middle ear fluid (MEF) are associated with reduced acute otitis media caused by Streptococcus pneumoniae (Spn). Nasopharyngeal samples were collected from agematched healthy children. The levels of pneumococcal specific to total immunoglobulin $\mathrm{G}(\mathrm{IgG})$ and $\operatorname{IgA}\left(\mathrm{ng} \mu \mathrm{g}^{-1}\right)$ were compared using the Mann-Whitney test. (a): Anti-PhtD, anti-PcpA, and anti-PlyD1 IgG; (b): anti-PhtD, anti-PcpA, and anti-PlyD1 IgA; tlgG and tlgA, total amount of $\lg \mathrm{G}$ and $\lg A$. Lines represent geometric means with $95 \%$ confidence intervals.
Although numerous studies have shown that serum antibodies rise following pneumococcal carriage, ${ }^{25,31-34}$ very limited information is available regarding mucosal antibody responses to respiratory bacterial pathogens in humans, especially infants and children. In large part, this is because reproducible collection of nasal secretions is challenging due to variability in acquiring mucus from the NP. Saliva is another source for study of mucosal immunity, and its collection is easier than NP samples. Zhang et al. ${ }^{35}$ found that children aged 2-12 years who were culture-positive for Spn in their NP had higher IgG but not IgA in serum and saliva to pneumococcal choline-binding protein A (CbpA) and Ply but not PsaA or PspA. However, NP mucosal antibody assays are more reproducible and reliable than saliva antibody levels, because saliva antibody levels are significantly influenced by many variables, such as saliva flow. ${ }^{36,37}$ There are no previous reports on mucosal antibody to PhtD, PcpA, and Ply in nasal secretions of young children by natural Spn NP colonization. The results of this study, along with our prior findings regarding serum antibody responses to Spn NP colonization in the same cohorts of subjects, ${ }^{25,31}$ clearly show that $S p n$ colonization is an immunizing event for both systemic- and mucosal-acquired immune responses. In addition, PhtD, PcpA, and Ply are highly immunogenic following natural exposure to Spn.

The elicitation of serum antibody following NP colonization by potential respiratory pathogens has been well documented in previous studies. Holmlund et al. ${ }^{32}$ reported an increase in serum antibody concentrations to PsaA and Ply in infants who were colonized with Spn. Simell et al. ${ }^{33}$ showed that children with prior positive NP cultures for $S p n$ had significantly higher serum anti-CbpA and anti-PhtD IgG titers. Prevaes et al. ${ }^{34}$ reported that colonization with Spn induced serum IgG against 14 pneumococcal proteins. Verkaik et al. ${ }^{38}$ found that children aged 6-24 months colonized by Staphylococcus aureus had
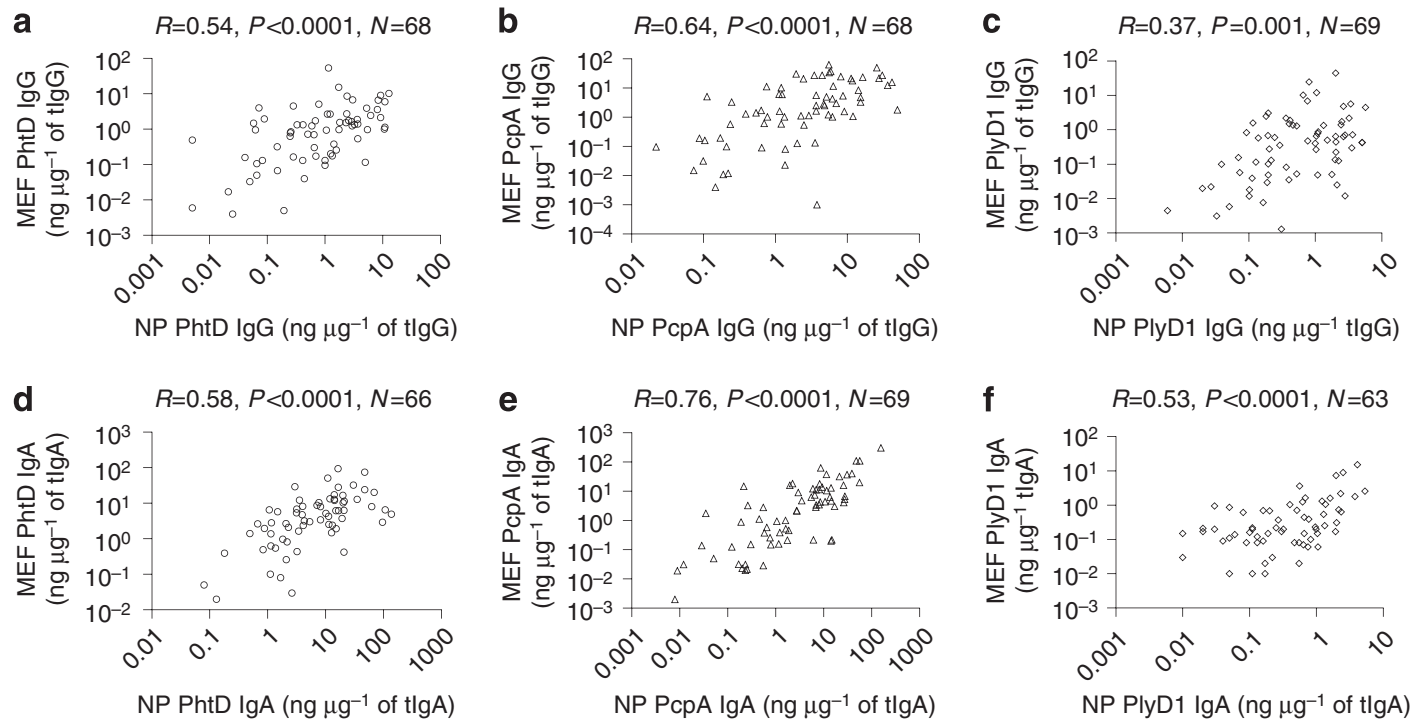

Figure 5 Correlations of antibody levels in the nasopharyngeal (NP) to middle ear fluid (MEF). Paired NP and MEF samples simultaneously collected from the same visit of the same children, and correlation of immunoglobulin $G$ (IgG) and IgA levels in NP to MEF was analyzed using the Spearman's coefficient. (a), Anti-PhtD IgG; (b), anti-PcpA IgG; (c), anti-PlyD1 IgG; (d), anti-PhtD IgA; (e), anti-PcpA IgA; (f), anti-PlyD1 IgA. 
higher serum IgG and IgA levels to a number of staphylococcal proteins than non-colonized children. Our research group previously reported that colonization with either Spn or $H$. influenzae elicited serum IgG and IgA responses to homologous bacterial species. ${ }^{25,28,31}$

The most important observation in this report is the identification of an association between higher mucosal antibody levels in NP and MEF to PhtD, PcpA, and PlyD1 and reduced risk of AOM. This implies the NP mucosal antibody levels to PhtD, PcpA, and Ply may have an important role in preventing Spn AOM in young children. Both IgG and IgA antibody levels in MEF are significantly associated with antibody levels detected in nasal secretions. Also of great relevance to vaccine design, we found that antibodies to these three vaccine candidate proteins did not require eradication of $S p n$ from the NP to positively impact the occurrence of $S p n$ $\mathrm{AOM}$. We hypothesize that protection from AOM occurred by reduction of the effective Spn inoculum (bacterial load) in the NP below a pathogenic threshold level. We are initiating a study to investigate this notion.

Our study has limitations. The colonization in our study was defined based on bacterial cultures at a moment in time when samples were collected. We did not determine NP bacterial or viral loads that have been proven to be critical variants influencing host immune responses. ${ }^{39}$ The antibody levels in this study were free antibody. The results might be influenced by bacterial load, bacterial agglutination with antibody, and soluble antigens (e.g., pneumolysin) released from Spn. The NP is the ecological niche for a variety of commensal microbiota as well as potential respiratory disease causing bacteria and viruses. ${ }^{40,41}$ Cross-reactive antigens that are expressed by other non-pneumococcal species (e.g., Streptococcus mitis) may influence the detected antibody titers. Capsule is regarded as a critical virulence factor for pathogenesis of pneumococcal infections. We did not measure mucosal anti-capsular titers and thus do not exclude the possibility that Spn AOM resulted from lack of capsular antibody to the $S p n$ serotypes. In addition, $5 \%$ of MEF and $20 \%$ of NP samples that had undetectable or very low total antibody levels were excluded in this study. As these samples distributed randomly to each age, colonization, and AOM group, and represented a small fraction of the total samples, the exclusion of those samples had limited influence on the conclusions of this study.

In summary, this is the first report to characterize the natural acquisition of mucosal NP and MEF antibody responses to $S p n$ candidate vaccine proteins $\mathrm{PhtD}, \mathrm{PcpA}$, and PlyD1 in young children who experience Spn colonization and AOM. We found that colonization by $S p n$ is positively associated with mucosal antibody levels to PhtD, PcpA, and PlyD1 proteins. Most importantly, we identified an association of higher mucosal antibody levels in the NP to PhtD, PcpA, and PlyD1 with reduced AOM caused by Spn. The results imply that NP mucosal antibody against PhtD, PcpA, and Ply are potential markers of anti-pneumococcal immunity and that these three proteins may elicit protection against $S p n$ mucosal infections, especially AOM.

\section{METHODS}

Study design. This study derives from a 5-year (2006-2011) prospective longitudinal evaluation of immunity to Spn and NTHi NP colonization and AOM in young children aged 6-24 months, supported by the US National Institute of Deafness and Communication Disorders. Healthy children without previous episodes of AOM were enrolled at 6 months of age from a middle class, suburban sociodemographic pediatric practice in Rochester, NY (Legacy Pediatrics). NP samples were obtained every 3-6 months prospectively from healthy children aged 6-24 months. When AOM occurred, tympanocentesis was performed to collect MEF and confirm the diagnosis of AOM, as previously described. ${ }^{42}$ At the time of an AOM diagnosis, NP and MEF samples were concurrently obtained. All children in this study who developed an AOM had common clinical symptoms of viral upper respiratory infection, such as cough, sore throat, runny nose, nasal congestion, headache, low-grade fever, and sneezing. All of the children received standard vaccinations, including the PCV-7 or PCV13 (Prevnar, Pfizer Pharmaceuticals, Collegeville, PA), at the appropriate age. The study was approved by the Institutional Review Board of the University of Rochester and Rochester General Hospital, and written informed consent was obtained from parents or guardians of all child subjects.

Sample collection. NP swab samples were obtained by inserting a cotton-tipped wire swab deeply into both nares. NP wash samples were obtained by instilling $1 \mathrm{ml}$ of sterile phosphate buffered saline and aspirating from both nares for antibody measurement. MEF samples for antibody measurement varied in quantity of material obtained from 50 to $250 \mu \mathrm{l}$, and the entire sample was added to $1 \mathrm{ml}$ of phosphate buffered saline ( $\mathrm{pH}$ 7.4). The NP wash samples and MEF samples were centrifuged at 3000 r.p.m. ( $1100 \mathrm{~g})$ at $4^{\circ} \mathrm{C}$ for $10 \mathrm{~min}$, and the supernatants were stored at $-80^{\circ} \mathrm{C}$ until use. NP swab samples and MEF samples were for microbiological culture, and NP wash samples and MEF samples were for antibody measurements.

Microbiology. Three potential bacterial pathogens, Spn, H. influenzae, and Moraxella catarrhalis, were isolated and identified according to tests of the eighth edition of the Manual of Clinical Microbiology. ${ }^{43}$

Quantitative enzyme-linked immunosorbent assay (ELISA) to detect pneumococcal antigen-specific IgG and IgA antibodies. $\mathrm{PhtD}, \mathrm{PcpA}$, and PlyD1-specific antibody IgG and IgA concentrations in the NP and MEF were determined by quantitative ELISA as previously described ${ }^{31}$ with modification. Briefly, 384-well highbinding microplates (Greiner Bio-One, Monroe, NC) were coated with $20 \mathrm{ng}$ of individual purified recombinant proteins in $20 \mu \mathrm{l}$ of coating buffer (bicarbonate, $\mathrm{pH} 9.6$ ) at $4{ }^{\circ} \mathrm{C}$ overnight and then blocked with $60 \mu \mathrm{l}$ of phosphate buffered saline containing $4.0 \%$ skim milk at $37^{\circ} \mathrm{C}$ for $1 \mathrm{~h}$. The samples were twofold serially diluted in $20 \mu \mathrm{l}$ phosphate buffered saline containing $4.0 \%$ skim milk at an initial dilution of 1:5 and incubated at room temperature for $1 \mathrm{~h}$, followed by an incubation with horseradish peroxidase-conjugated anti-human IgG or IgA (Bethyl Laboratories, Montgomery, TX). The reaction products were developed with TMB Microwell Peroxidase Substrate System (KPL, Gaithersburg, MD), stopped by $20 \mu \mathrm{l}$ of $1.0 \mathrm{M}$ phosphoric acid, and read by an automated ELISA reader (Molecular Devices, Sunnyvale, CA) using a $450 \mathrm{~nm}$ filter. A standard curve was generated using a fourparameter logistic-log function and a reference human serum containing known specific antibody concentrations to corresponding individual antigens. Antigen-specific IgG and IgA levels against the three pneumococcal antigens in the reference serum were quantified using Human IgA and IgG ELISA Quantitation Sets (Bethyl Laboratories, Montgomery, TX) as described previously. ${ }^{31}$ The assay lower limits of detection were $2.4 \mathrm{ng} \mathrm{ml}^{-1}$ for anti-PhtD IgG, $0.18 \mathrm{ng} \mathrm{ml}^{-1}$ for anti-PhtD IgA; $2.2 \mathrm{ng} \mathrm{ml}^{-1}$ for anti-PcpA IgG, $0.28 \mathrm{ng} \mathrm{ml}^{-1}$ for anti-PcpA IgA, $1.0 \mathrm{ng} \mathrm{ml}^{-1}$ for anti-PlyD1 IgG, and $0.22 \mathrm{ng} \mathrm{ml}^{-1}$ for anti-PlyD1 IgA. An internal control was included in each plate for each antigen and the inter-assay coefficient of variation 
was $\leq 20 \%$ for all antigens and secondary antibody combinations. For the purpose of statistical analysis, undetectable samples were arbitrarily assigned a value equivalent to half the lower limit of detection of corresponding specific antibodies. ELISAs were fully validated according to ICH Guidance and performed in a GLP laboratory.

To correct for differential dilution effects that occurred during the NP wash and MEF sample collection, total IgG and IgA were determined using the Human IgG and IgA ELISA Quantitation Kits (Bethyl Laboratories) according to the manufacturer's protocol. Mucosal antigen-specific IgG and IgA in NP and MEF samples were then corrected according to the total IgG or IgA in the sample. Results were expressed as a ratio of specific IgG to total IgG or specific IgA to total IgA in the same sample (ng $\mu \mathrm{g}^{-1}$ ) as described previously. ${ }^{44,45}$ Samples with a total IgG or IgA $<0.05 \mu \mathrm{g} \mathrm{ml}^{-1}$ were excluded, because in preliminary studies we determined that such samples were from children with a difficult or failed sampling process and had undetectable antigen-specific antibodies. In all 5\% of MEF and $20 \%$ of NP samples were excluded in this study according to this criterion.

Statistics. Statistical analysis was performed with the R Project version 2.13.2 (www.r-project.org/). Antibody levels were expressed as GM with $95 \%$ CIs of ratios of specific to total IgG or IgA. The associations between antibody levels and AOM occurrences and ages were analyzed using Generalized Estimating Equations (GEE) to fit a repeatedmeasures logistic regression models. The AOM ORs between the 95th and 5th percentile antibody level were estimated using GEE models with AR1 subject level correlation and quadratic terms. The paired NP and MEF samples that were collected from the same AOM visits of the same subject were used to analyze the correlation between antibody levels in the NP and MEF. Because antibodies were found to correlate significantly with age, to control for a spurious latent correlation, antibody levels were adjusted for age using a GEE model, assuming a within-subject auto-regressive correlation. Antibody levels were subject to Box-Cox power transformations, and age was log-transformed. ${ }^{46}$ CIs and levels of significance for (age-adjusted) antibody correlations were estimated using a bootstrap procedure with subjectlevel resampling. Antibody levels between age-matched groups (colonization, non-colonization, Spn AOM, non-Spn AOM groups) were compared using the non-parametric two-tailed Mann-Whitney test with GraphPad Prism 6.0 (La Jolla, CA). $P<0.05$ was considered to indicate statistical significance.

\section{ACKNOWLEDGMENTS}

This study was supported by an investigator-initiated grant from Sanofi pasteur and NIH NIDCD RO1 08671. We thank the nurses and staff of Legacy Pediatrics and the collaborating pediatricians from Sunrise Pediatrics, Westfall Pediatrics, Lewis Pediatrics and Long Pond Pediatrics and the parents who consented and the children who participated in this long and challenging study. We also thank Dr Anthony Almudevar (Department of Biostatistics and Computational Biology, University of Rochester Medical Center) for data satatsitical analysis, Jill Mangiafesto, Czup, Katerina, Virginia Judson and Emily Newman, for assistant with ELISA, and Dr Robert Zagursky for reviewing, editing and commenting the manuscript.

\section{DISCLOSURE}

The authors declare no conflict of interest.

c 2015 Society for Mucosal Immunology

\section{REFERENCES}

1. Lynch, J.P. 3rd \& Zhanel, G.G. Streptococcus pneumoniae: epidemiology and risk factors, evolution of antimicrobial resistance, and impact of vaccines. Curr. Opin. Pulm. Med. 16, 217-225 (2010).

2. Pavia, M., Bianco, A., Nobile, C.G., Marinelli, P. \& Angelillo, I.F. Efficacy of pneumococcal vaccination in children younger than 24 months: a metaanalysis. Pediatrics 123, e1103-e1110 (2009).
3. van der Linden, M., Reinert, R.R., Kern, W.V. \& Imohl, M. Epidemiology of serotype 19A isolates from invasive pneumococcal disease in German children. BMC Infect. Dis. 13, 70 (2013).

4. Calix, J.J. et al. Biochemical, genetic, and serological characterization of two capsule subtypes among Streptococcus pneumoniae serotype 20 strains: discovery of a new pneumococcal serotype. J. Biol. Chem. 287, 27885-27894 (2012)

5. Ruckinger, S., van der Linden, M., Reinert, R.R. \& von Kries, R. Efficacy of 7 -valent pneumococcal conjugate vaccination in Germany: an analysis using the indirect cohort method. Vaccine 28, 5012-5016 (2010).

6. Besser, T.E., McGuire, T.C., Gay, C.C. \& Pritchett, L.C. Transfer of functional immunoglobulin $\mathrm{G}(\mathrm{IgG})$ antibody into the gastrointestinal tract accounts for lgG clearance in calves. J. Virol. 62, 2234-2237 (1988).

7. Pichichero, M.E. \& Casey, J.R. Emergence of a multiresistant serotype 19A pneumococcal strain not included in the 7 -valent conjugate vaccine as an otopathogen in children. JAMA 298, 1772-1778 (2007).

8. Kaplan, S.L. et al. Serotype 19A is the most common serotype causing invasive pneumococcal infections in children. Pediatrics 125, 429-436 (2010).

9. Moffitt, K.L. \& Malley, R. Next generation pneumococcal vaccines. Curr. Opin. Immunol. 23, 407-413 (2011).

10. Jambo, K.C., Sepako, E., Heyderman, R.S. \& Gordon, S.B. Potential role for mucosally active vaccines against pneumococcal pneumonia. Trends Microbiol. 18, 81-89 (2010).

11. Vila-Corcoles, A. et al. Effectiveness of the 23-valent polysaccharide pneumococcal vaccine against invasive pneumococcal disease in people 60 years or older. BMC Infect. Dis. 10, 73 (2010).

12. Darrieux, M., Goulart, C., Briles, D. \& Leite, L.C. Current status and perspectives on protein-based pneumococcal vaccines. Crit. Rev. Microbiol. 7, 1-11 (2013).

13. Feldman, C. \& Anderson, R. Review: current and new generation pneumococcal vaccines. J. Infect. 69, 309-325 (2014).

14. Seiberling, M. et al. Safety and immunogenicity of a pneumococcal histidine triad protein $\mathrm{D}$ vaccine candidate in adults. Vaccine $30,7455-$ 7460 (2012).

15. Bologa, M. et al. Safety and immunogenicity of pneumococcal protein vaccine candidates: monovalent choline-binding protein $A(P c p A)$ vaccine and bivalent PcpA-pneumococcal histidine triad protein $D$ vaccine. Vaccine 30, 7461-7468 (2012).

16. Kamtchoua, T. et al. Safety and immunogenicity of the pneumococcal pneumolysin derivative PlyD1 in a single-antigen protein vaccine candidate in adults. Vaccine 31, 327-333 (2013).

17. Bersch, B. et al. New insights into histidine triad proteins: solution structure of a Streptococcus pneumoniae PhtD domain and zinc transfer to AdcAll. PLoS One 8, e81168 (2013).

18. Khan, M.N., Sharma, S.K., Filkins, L.M. \& Pichichero, M.E. PcpA of Streptococcus pneumoniae mediates adherence to nasopharyngeal and lung epithelial cells and elicits functional antibodies in humans. Microbes Infect. 14, 1102-1110 (2012).

19. Barocchi, M.A., Censini, S. \& Rappuoli, R. Vaccines in the era of genomics: the pneumococcal challenge. Vaccine 25, 2963-2973 (2007).

20. Plumptre, C.D., Ogunniyi, A.D. \& Paton, J.C. Vaccination against Streptococcus pneumoniae using truncated derivatives of polyhistidine triad protein D. PLoS One 8, e78916 (2013).

21. Verhoeven, D., Perry, S. \& Pichichero, M.E. Contributions to protection from Streptococcus pneumoniae infection using the monovalent recombinant protein vaccine candidates PcpA, PhtD, and PlyD1 in an infant murine model during challenge. Clin. Vaccine Immunol. 21, 1037-1045 (2014)

22. Verhoeven, D., Xu, Q. \& Pichichero, M.E. Vaccination with a Streptococcus pneumoniae trivalent recombinant PcpA, PhtD and PlyD1 protein vaccine candidate protects against lethal pneumonia in an infant murine model. Vaccine 32, 3205-3210 (2014).

23. Glover, D.T., Hollingshead, S.K. \& Briles, D.E. Streptococcus pneumoniae surface protein PcpA elicits protection against lung infection and fatal sepsis. Infect. Immun. 76, 2767-2776 (2008).

24. Mann, B. et al. Broadly protective protein-based pneumococcal vaccine composed of pneumolysin toxoid-CbpA peptide recombinant fusion protein. J. Infect. Dis. 209, 1116-1125 (2014). 
25. Pichichero, M.E. et al. Antibody response to Streptococcus pneumoniae proteins PhtD, LytB, PcpA, PhtE and Ply after nasopharyngeal colonization and acute otitis media in children. Hum. Vaccin. Immunother. 8, 799-805 (2012).

26. Heikkinen, T. \& Chonmaitree, T. Importance of respiratory viruses in acute otitis media. Clin. Microbiol. Rev. 16, 230-241 (2003).

27. Kaur, R., Kim, T., Casey, J.R. \& Pichichero, M.E. Antibody in middle ear fluid of children originates predominantly from sera and nasopharyngeal secretions. Clin. Vaccine Immunol. 19, 1593-1596 (2012).

28. Pichichero, M.E. et al. Antibody response to Haemophilus influenzae outer membrane protein D, P6, and OMP26 after nasopharyngeal colonization and acute otitis media in children. Vaccine 28, 7184-7192 (2010).

29. Syrjanen, R.K., Kilpi, T.M., Kaijalainen, T.H., Herva, E.E. \& Takala, A.K. Nasopharyngeal carriage of Streptococcus pneumoniae in Finnish children younger than 2 years old. J. Infect. Dis. 184, 451-459 (2001).

30. Sato, S. \& Kiyono, H. The mucosal immune system of the respiratory tract. Curr. Opin. Virol. 2, 225-232 (2012).

31. Xu, Q. \& Pichichero, M.E. Co-colonization by Haemophilus influenzae with Streptococcus pneumoniae enhances pneumococcal-specific antibody response in young children. Vaccine 32, 706-711 (2014).

32. Holmlund, E., Quiambao, B., Ollgren, J., Nohynek, H. \& Kayhty, H. Development of natural antibodies to pneumococcal surface protein $\mathrm{A}$, pneumococcal surface adhesin A and pneumolysin in Filipino pregnant women and their infants in relation to pneumococcal carriage. Vaccine $\mathbf{2 4}$ 57-65 (2006).

33. Simell, B. et al. Pneumococcal carriage and acute otitis media induce serum antibodies to pneumococcal surface proteins CbpA and PhtD in children. Vaccine 27, 4615-4621 (2009).

34. Prevaes, S.M. et al. Nasopharyngeal colonization elicits antibody responses to staphylococcal and pneumococcal proteins that are not associated with a reduced risk of subsequent carriage. Infect. Immun. $\mathbf{8 0}$, 2186-2193 (2012)
35. Zhang, Q et al. Serum and mucosal antibody responses to pneumococcal protein antigens in children: relationships with carriage status. Eur. J. Immunol. 36, 46-57 (2006).

36. Bellussi, L., Cambi, J. \& Passali, D. Functional maturation of nasal mucosa: role of secretory immunoglobulin A (SlgA). Multidiscip. Respir. Med. 8, 46 (2013).

37. Brandtzaeg, P. Do salivary antibodies reliably reflect both mucosal and systemic immunity? Ann. NY Acad. Sci. 1098, 288-311 (2007).

38. Verkaik, N.J. et al. Induction of antibodies by Staphylococcus aureus nasal colonization in young children. Clin. Microbiol. Infect. 16, 1312-1317 (2010).

39. Franz, A. et al. Correlation of viral load of respiratory pathogens and coinfections with disease severity in children hospitalized for lower respiratory tract infection. J. Clin. Virol. 48, 239-245 (2010).

40. Bosch, A.A., Biesbroek, G., Trzcinski, K., Sanders, E.A. \& Bogaert, D. Viral and bacterial interactions in the upper respiratory tract. PLoS Pathogens $\mathbf{9}$, e1003057 (2013).

41. Pettigrew, M.M. et al. Viral-bacterial interactions and risk of acute otitis media complicating upper respiratory tract infection. J. Clin. Microbiol. 49, 3750-3755 (2011).

42. Pichichero, M.E. \& Wright, T. The use of tympanocentesis in the diagnosis and management of acute otitis media. Curr. Infect. Dis. Rep. 8, 189-195 (2006).

43. Murray, P.R., BEPfaller, M.A., Jorgensen, J.H. \& Yolken, R.H. (eds). Manual of Clinical Microbiology, 8th edn. ASM Press, Washington DC (2003).

44. Hammitt, L.L. et al. Kinetics of viral shedding and immune responses in adults following administration of cold-adapted influenza vaccine. Vaccine 27, 7359-7366 (2009).

45. Durrer, P. et al. Mucosal antibody response induced with a nasal virosomebased influenza vaccine. Vaccine 21, 4328-4334 (2003).

46. Venables, W.N. \& Ripley, B.D. Modern Applied Statistics with S. (Springer, New York, NY, USA, 2010). 